Desi gn and opt i mzat i on of an advanced ti me- of- $\mathrm{fl}$ i ght neut ron spect ronet er for deut er i um pl asmas of the I arge hel i cal devi ce

\begin{tabular}{|l|l|}
\hline $\begin{array}{l}\text { jour nal or } \\
\text { publ i cat } \mathrm{i} \text { on } \mathrm{t} \text { i t l e }\end{array}$ & Revi ew of Sci ent i f i c I nst rument s \\
\hline vol une & 92 \\
\hline number & 5 \\
\hline page $\mathrm{r}$ ange & 053547 \\
\hline year & $2021-05-24$ \\
\hline URL & ht t p: //hdl . handl e. net /10655/00012771 \\
\hline
\end{tabular}




\title{
Design and Optimization of an advanced time-of-flight neutron spectrometer for deuterium plasmas of the Large Helical Device ${ }^{\text {a) }}$
}

\author{
Yimo Zhang, ${ }^{1}$ Lijian Ge, ${ }^{1}$ Zhimeng Hu, ${ }^{1,3}$ Jiaqi Sun, ${ }^{1}$ Xiangqing Li,${ }^{1}$ Kunihiro Ogawa, ${ }^{2}$ Mitsutaka \\ Isobe,${ }^{2}$ Siriyaporn Sangaroon, ${ }^{2}$ Longyong Liao, ${ }^{1}$ Danke Yang, ${ }^{1}$ Giuseppe Gorini, ${ }^{3,4}$ Massimo \\ Nocente, ${ }^{3,4}$ Marco Tardocchi, ${ }^{4}$ Tieshuan Fan $\left.{ }^{1, b}\right)$ \\ ${ }^{1}$ State Key Laboratory of Nuclear Physics and Technology, Peking University, Beijing 100871, China \\ ${ }^{2}$ National Institute for Fusion Science, 322-6 Oroshi-cho, Toki 509-5292, Japan \\ ${ }^{3}$ Dipartimento di Fisica 'G. Occhialini', Università degli Studi di Milano-Bicocca, Milano 20126, Italy \\ ${ }^{4}$ Institute for Plasma Science and Technology, National Research Council, Milan 20125, Italy
}

(Presented XXXXX; received XXXXX; accepted XXXXX; published online XXXXX)

(Dates appearing here are provided by the Editorial Office)

A time-of-flight neutron spectrometer based on the TOFED concept has been designed and is under development for the Large Helical Device (LHD). It will be the first advanced neutron spectrometer to measure the 2.5 MeV D-D neutrons (DDN) from helical/stellarator plasmas. The main mission of the new TOFED is to study the energetic ion behaviours in helical plasmas, which includes both deuterons from the auxiliary heating systems and tritons born from the $\mathrm{d}+\mathrm{d}$ fusion reactions. The latter are measured by the triton burn-up neutrons (TBN) and the new spectrometer, unlike the original TOFED in the EAST tokamak, has been optimized for this scope. Based on Monte Carlo simulation by a GEANT4 model, the energy resolutions for DDN and TBN are $6.6 \%$ and $9.0 \%$, respectively. The new TOFED is expected to be installed in the basement under the LHD hall, and shares the collimator with one channel of the neutron camera, which defines a vertical line-of-sight. The distance from the first scintillators of the new TOFED to the equatorial plane of LHD plasmas is about 15.5 meters. When projected to the neutron rates that are typically obtained in LHD deuterium plasmas, (order of $10^{15} \mathrm{n} / \mathrm{s}$ with neutral beam injection) we expect to obtain the DDN and TBN counting rates of about $250 \mathrm{kHz}$ and $250 \mathrm{~Hz}$, respectively. This will make the simultaneous study of fast deuterons and fusion born tritons with neutron spectroscopy possible on time scales of few seconds, for the first time in helical/stellarator plasmas.

\section{INTRODUCTION}

Neutrons produced from D-D and D-T reactions carry the information about the kinematic state of fusion fuels in plasmas. Among magnetic confined fusion devices like tokamaks, the neutron diagnostics have been widely adopted $^{1}$. ParticularlyIn particular, the advanced Neutron Emission Spectroscopy (NES) diagnostics, such as the $\mathrm{MPR}^{2}$ and the time-of-flight neutron spectrometer TOFOR ${ }^{3}$ at the JETJoint European Torus, have been proved asproved to be powerful tools are amonginstruments to understand the behavior of energetic particles ${ }^{4-8}$ in fusion experiments, which is one of the most concerned issues in the ITER burning plasma physiesof relevance for burning plasma devices, such as ITER $^{9,10}$. With regard to the present stellarators and helical devices, the Large Helical Device (LHD) has firstly initiatedstarted its first deuterium experimental eampaigns fromcampaign in 2017 , in order to explore the related fusion plasma physics and engineering

${ }^{\text {a) }}$ Published as part of the Proceedings of the Virtual $23^{\text {rd }}$ Topical Conference on High-Temperature Plasma Diagnostics (HTPD) hosted by Los Alamos National Laboratory.

b) Author to whom correspondence should be addressed:

tsfan@pku.edu.cn problems ${ }^{11}$ challenges ${ }^{11}$. There are already rather comprehensive neutron diagnostics at the LHD, including neutron flux monitors, neutron activation systems, a vertical neutron camera, and scintillating-fiber detectors, which all operate with good performance during the recent experiments ${ }^{12}$. The recorded neutron emission rates at the LHD reach more than $10^{15} \mathrm{n} / \mathrm{s}^{13}$, due to a large number of fast ions, mainly generated from the external power by the auxiliary heating system.

For studying the fast ion behavioursIn order to advance our knowledge on the fast ion behaviour in the helical fusion plasmas, a new advanced time-of-flight neutron spectrometer based on the TOFED (Time-Of-Flight Enhanced Diagnostics) concept ${ }^{14}$ is projected towill be developed at the LHD, which will and it will be the first advanced neutron spectrometer for the a helical/stellarator plasmas. The TOFED neutron spectrometer, which originates from the TOFOR concept but retains $\underline{\text { has an }}$ improved the optimized-efficiency and energy resolution due to its double-ring secondary scintillator array, has been developed and put into successful operations at the EAST tokamak for diagnosing thestudies of fast ions from the NBI (Neutral Beam Injection) and ICRF (Ion Cyclotron Resonance Frequency) heating plasmas ${ }^{15}$ systems $^{-18}$. Moreover, time-resolved triton burn-up diagnosis have been 


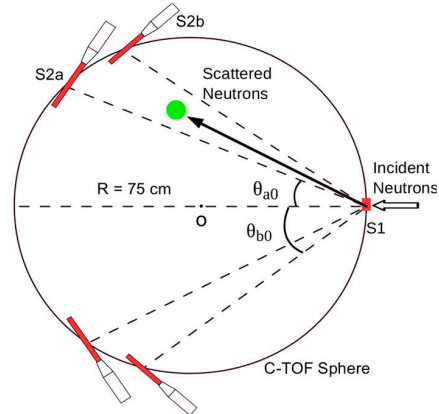

Fig. 1 The principle of the TOFED concept is exhibitedshown. The primary scintillators $\mathrm{S} 1$ and the secondary scintillators $\mathrm{S} 2$ are arranged on the surface of the CTOF sphere. If When the collimated neutron flux perpendicularly hit the S1 scintillator, some neutrons will be deflected to the S2 detectors and their interaction recorded. Under these circumstances, the the protons inside the S1 and then seatter to the S2, the time-of-flight of scattering the scattered neutrons are-is only determined by the incident neutrons energy ${ }_{2}$ regardless of the different flight routepath scattered neutrons may follow from S1 to S2.

conducted by the measurements of the $14 \mathrm{MeV}$ D-T neutrons, such as using the scintillating-fiber detectors. According to the recent experimental results at LHD, the Triton Burnup Neutrons (TBN) yields exceed $10^{12} \mathrm{n} / \mathrm{s}$, up to $0.5 \%$ of the D-D neutrons (DDN) ${ }^{19}$. It will be an attractive application of the TOFED-type neutron spectrometer at the LHD if this new instrument can appropriately respond to both DDN and TBN from the helical fusion plasmas.

In this paper, the new TOFED neutron spectrometer at the LHD will beis described. Section II briefly introduces the basic principle of the neutron time-of-flight measurement. In section III, the TOFED-type spectrometer concept at the LHD is discussed in detail. These results are summarized in the last section.

\section{TIME-OF-FLIGHT PRINCEPLEPRINCIPLE}

The time-of-flight (TOF) neutron spectrometer consists of two sets of scintillators, see Figure 1. Assumed Assuming that all the scintillators are tangential to a sphere (with a radius $R$ ) at their centers, the collimated neutrons hit the primary scintillators (S1) at the center along the diameter of the sphere. If one neutron with the energy $E_{n}$ eollides with one proton insidehas a single scattering collision with a proton in $\mathrm{S} 1$, i.e. for just one time by an elastic seattering process $n+p \rightarrow p_{r}+n^{\prime}$, in which the neutron scattering angle is $\theta$, the flight time $t_{t \theta f}$ for the scattering neutron to collide again inside the secondary scintillator (S2) can derived fromand then reaches the S2 scintillators, we have

$$
\begin{aligned}
& t_{\text {tof }}=\frac{2 R \cos \theta}{v_{n}^{\prime}}, \\
& E_{n}^{\prime}=E_{n} \cos ^{2} \theta=\frac{m_{n} v_{n}^{\prime 2}}{2},
\end{aligned}
$$

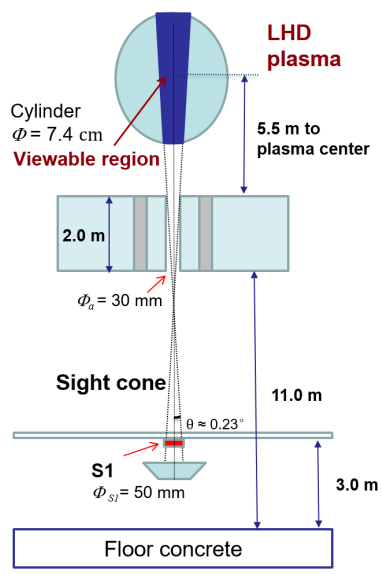

Fig. 2 The LHD-TOFED is expected to be installed under the LHD plasmas. A hole with a radius of $30 \mathrm{~mm}$ through the 2-meters-thick basement is used as the collimator and radiation shielding. The distance from the equatorial plane of LHD plasmas to the S1 scintillator of LHD-TOFED is at least $15.5 \mathrm{~m}$. This collimator, which is originally used by the vertical neutron camera, defines a line-ofsight with a divergence of no more than $0.23^{\circ}$, and gives LHDTOFED a viewable region which can be approximatively regarded as a cylinder with a radius of about $7.4 \mathrm{~cm}$.

Where $\theta$ is the scattering angle, $v_{n}^{\prime}$ means-is the velocity of the neutron after the first scattering, and $m_{n}$ is the mass of the neutron and thof denotes the time of flight. Therefore, energies of the incident neutron and the recoil proton inside the $\mathrm{S} 2, E_{n}$ and $E_{p}$, ean be denoted asare obtained as

$$
\begin{aligned}
& E_{n}=\frac{2 m_{n} R^{2}}{t_{\text {tof }}^{2}} \\
& E_{p}=E_{n} \sin ^{2} \theta=\frac{2 m_{n} R^{2}}{t_{\text {tof }}^{2}} \sin ^{2} \theta
\end{aligned}
$$

which-This implies that means that the seattering neutronsscattered neutrons born from incoming neutrons with the same energy $\mathrm{E}_{\mathrm{n}}$ take the same time towill spend equal time to reach the identical spherical surface despite of

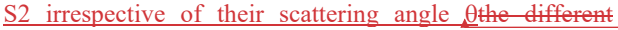
scattering angle. This is the so-called constant time-of-flight (CTOF) sphere principle. But the nNeutrons scattering multiple times inside the S1 would not obeywill however break this principle the CTOF principle ${ }^{20}$. As shown in Fig. 1 , the two rings of the TOFED's S2 scintillators cover a certain range of neutron scattering angles, thus determining the $E_{p}$ collected by the $S 1$ a range for the scattered proton energy Ep that can be released in S1, given En. The effective neutron events can thus be discriminated from the multiple events or carbon scattering events by the time-of-flight and recoiled proton energy, which constitutes the fundament principle of the double kinematic selection (DKS) method $^{15,21}$. In the TOFED case, the radius of the CTOF is
書式を変更：フォント：Symbol

書式を変更：下付き

書式を変更：下付き

書式を変更：フォント：Symbol 
$1.5 \mathrm{~m}$, and the range of the scattering angles is $20^{\circ}<\theta<31^{\circ}$ for $\mathrm{S} 2 \mathrm{a}$ and $31^{\circ}<\theta<40^{\circ}$ for $\mathrm{S} 2 \mathrm{~b}$, respectively.

\section{TOFED-TYPE SPECTROMETER AT THE LHD}

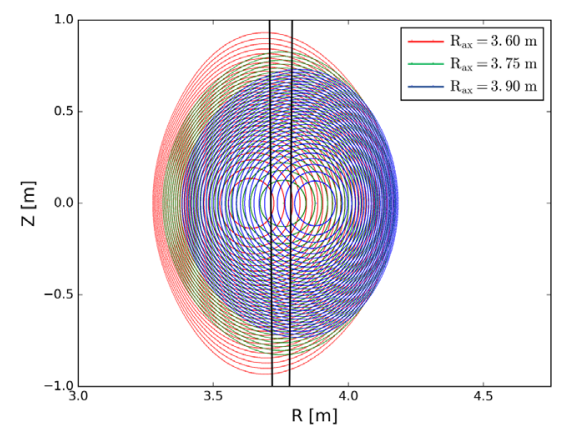

Fig. 3 The line-of-sight of the LHD-TOFED gives an approximately cylindrical viewable-viewing region (between the black lines). For the three typical magnetic configurations $\left(\mathrm{R}_{\mathrm{ax}}=3.6 \mathrm{~m}\right.$ in red, $\mathrm{R}_{\mathrm{ax}}=3.75 \mathrm{~m}$ in green, and $\mathrm{R}_{\mathrm{ax}}=3.9 \mathrm{~m}$ in blue), the LHD-TOFED can always collect the fusion neutrons from the helical plasmas core.

The LHD is one of the largest superconducting helical device with a major radius/ minor radius of $3.9 \mathrm{~m} / \sim 0.6 \mathrm{~m}$. Its NBI heating system consists of three tangentiallyinjected neutral beams and two perpendicularly-injected neutral beams with maximum beam energy of around 180 $\mathrm{keV}$ and $60 \mathrm{keV}$, respectively, and the total NBI power can be up to $30 \mathrm{MW}$. Besides, the ICRF system with total power of about $6 \mathrm{MW}$ is also a main heating source at the $\mathrm{LHD}^{12}$. Analyzing the neutron spectra to obtain the kinematic states of the fuel ions, the TOFED-type neutron spectrometer (mentioned as LHD-TOFED in the later sections) is primarily aimed at the research of the substantialat studying fast ions induced-produced by these auxiliary heating in the LHD plasmas.

\section{A. Line of sight}

The defined-LHD-TOFED is expected to be installed in the basement under the LHD hall, and shares one of the collimators belonged belonging to the middle channel of the vertical neutron camera, with a view line that passes going through the a concrete shielding. The distance from the equational plane of LHD plasma to the S1 scintillator of LHD-TOFED is at least $15.5 \mathrm{~m}$, as drawn in Fig. 2. This collimator with a radius of $30 \mathrm{~mm}$ defines a line-of-sight with a divergence of no more than $0.23^{\circ}$, and gives LHDTOFED a viewle viewing region which ean beis approximatively regarded as - a cylinder with a radius of about $7.4 \mathrm{~cm}$. Despite that the rotational LHD plasma, the pThe plasma profile observed by the LHD-TOFED ean be treated as anis approximately an ellipse of which major axis is vertical. with a vertical major axis.
In LHD, Tthe magnetic configuration can be shifted eutwardly or inwardlyoutward or inward as illustrated in Fig. 3. During the typical operation regime at the LHD, the radii of the magnetic axis usually range from $3.6 \mathrm{~m}$ to 3.9 $\mathrm{m}$. On this conditionIn these conditions, the line-of-sight of the new instrument (black line) can always ensure the observation of a collimated line-integrated neutron flux from the helieal plasma coreplasma core of the helical plasma.

TABLE I. The main technical parameters of the APV8104-14 digitizer.

\begin{tabular}{|c|c|c|c|c|c|}
\hline \hline $\begin{array}{c}\text { Sampling } \\
\text { rate }\end{array}$ & $\begin{array}{c}\text { Number of } \\
\text { channels }\end{array}$ & $\begin{array}{c}\text { Bit } \\
\text { number }\end{array}$ & $\mathrm{V}_{\mathrm{pp}}$ & bandwidth & RAM \\
\hline $1 \mathrm{Gs} / \mathrm{s}$ & 4 & 14 & $\pm 3 \mathrm{~V}$ & $500 \mathrm{MHz}$ & $2 \mathrm{~GB}$ \\
\hline
\end{tabular}

\section{B. Data Acquisition}

The Pdigital DAQ (data acquisition) system envisaged for the LHD-TOFED is based on the-compat digitizers is eompact and able tothat can acquire various information about the nuclear events at the same time, such as the triggering time and pulse height of the neutron pulsesmeasure the pulse height and time information of neutron scattering events in S1 and S2. In recent experiments, various - several NES diagnostics at the tokamaksin tokamaks around the world have upgraded the electronics through the employment of digitizers ${ }^{22-24}$. We have chosen a candidate digitizer (The APV8104-14 from Techno AP 7 as been chosen as the reference for the LHDTOFED. Its main features and parameters are listed in TABLE I. The communication between this type of digitizer and the computer is established by the TCP/IP protocol. The digital DAQ system can be fairly tolerable of counting rates in orderswork at counting rates up to-of $1 \mathrm{MHz}$ at the LHD due to the 2 GB RAM and the data transmission by optical fibers. It is validated that the A sampling rate of $1 \mathrm{G}$ samples/s satisfies the requirements of sub-nanosecond level precision for neutron time-of-flight measurements, combined with the CFD (Constant Fraction Discrimination) method in the off-line data processing ${ }^{18,22}$. The mix mode for data acquisition can collect both the pulse shape and triggering time, making it possible to apply the DKS method to improving improve the signal-to-noise of the neutron time-of-flight spectra. More dĐetailed work-tests on of the digital DAQ system will be conducted in the future.

\section{Response to DDN and TBN}



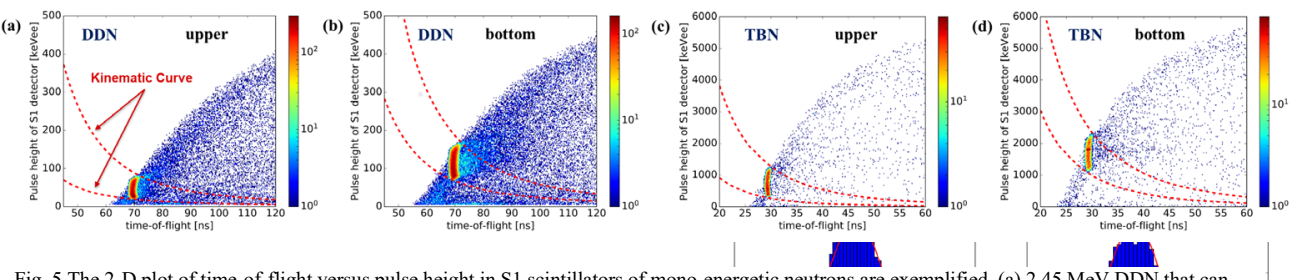

Fig. 5 The 2-D plot of time-of-flight versus pulse height in S1 scintillators of mono-energetic neutrons are exemplified. (a) $2.45 \mathrm{MeV}$ DDN that can be collected by the upper-ring S2 scintillators. (b) $2.45 \mathrm{MeV}$ DDN that can be collected by the bottom-ring S2 scintillators. (c) $14 \mathrm{MeV}$ TBN that can be collected by the upper-ring S2 scintillators. (d) $14 \mathrm{MeV}$ TBN that can be collected by the bottom-ring S2 scintillators. The effective singlescattering neutron events concentrate between the kinematic curves (red dotted line).

Since the LHD has provided high neutron rate comparable to that at of large tokamaks like JET, considerable triton burnup neutrons buld beare emitted from the its helical plasmas. A Monte Carlo model is developed by means-of the-neutron transport simulation from the plasma to the LHD-TOFED has been developed using the GEANT code $^{25}$ for the LHD-TOFED. In the simulation, the scintillators of both S1 (EJ228) and S2 (EJ200) keep unchanged from the originatare the same as those of the original TOFED. The performances of two kinds type of scintillators are have been previously investigated and their light output function is calibrated by $\underline{\text { s }}$ known from calibration experiments at he acelerater neutronsneutron accelerators ${ }^{15,16}$. Its-Their responses to both

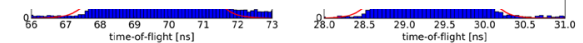

single-time scattered on neutron inside the S1 obey the Fig. 4 The simulated time-of-flight spectra for mono-energetic (a) $2.45 \mathrm{MeV}$ and (b) $14 \mathrm{MeV}$ neutrons are shown. The Gaussian fitting curves are plotted in red.

eequation (4) and depends on the range of the neutron scattering angle, and thus the scintillation light output induced by these protons are restricted by the scattering angles of neutrons. In the 2D figure of time-of-flight versus pulse height of the protons in the S1, pictured shown in Fig. 5 , we find that the effective-single scattering neutron events concentrate between the two kinematic curves. Thesewhich depend only on the finite scattering angle range

TABLE II. The energy resolutions of LHD-TOFED for DDN and TBN.

\begin{tabular}{|c|c|c|c|c|c|}
\hline \hline $\begin{array}{c}E_{n} \\
{[\mathrm{MeV}]}\end{array}$ & $\begin{array}{c}t_{\text {tof }} \\
{[\mathrm{ns}]}\end{array}$ & $\begin{array}{c}\text { FWHM from } \\
\text { geometry[ns] }\end{array}$ & $\begin{array}{c}\text { FWHM from } \\
\text { light propagation [ns] }\end{array}$ & $\begin{array}{c}\text { FWHM from } \\
\text { DAQ [ns] }\end{array}$ & $\begin{array}{c}\text { Energy resolution } \\
{[\%]}\end{array}$ \\
\hline 2.45 & 69.5 & 2.09 & 0.6 & 0.5 & 6.6 \\
14.0 & 29.3 & 1.88 & 0.6 & 0.5 & 9.0 \\
\hline
\end{tabular}

the-2.5 MeV DDN and the $14 \mathrm{MeV}$ TBN emission are-has been also evaluated.

The overall geometry of the scintillator array of LHDTOFED is responsible for a Gaussian shape broadening of the time of flight spectrum resulting from a collimated beam of $2.5 \mathrm{MeV}$ monoenergetic neutrons as input. Collimated mono-energetic neutrons bring a Gaussian-shaped time-offlight spectrum in the simulation due to the geometry of the seintillator array in the LHD-TOFED. The fitting resultsBy fitting the simulations results, we obtan show that the DDN peak on $t_{\text {tof }}$-axis is located at around $69.5 \mathrm{~ns}$, while the TBN peak at $29.3 \mathrm{~ns}$, as shown in Fig. 4. The FHWMs for DDN and TBN spectra are $2.09 \mathrm{~ns}$ and $1.88 \mathrm{~ns}$, respectively. Accounting the contributions By also taking into account the contribution from-by the digital DAQ system and light transmission from the scintillators to the photo multiplier tube, the total energy resolutions are determined to beef the integrated neutron spectrometer would be about $6.6 \%$ for DDN and $9.0 \%$ for TBN, respectively. The-More details on the contribution of each term to the overall of energy resolutions of the instrument are given in TABLE II.

As far as the energy released by single scattered protons in $\mathrm{S} 1$ is concerned, this is determined by Energy of protons determined by the solid angle covered by S2.enly rely on the fixed range of the scattering angles due to the finite solid angle covered by the $S 2$. Therefore, a lot of multiplemost of the multiple scattering seattering events $s_{2}$ or those due single scattering on carbon-scattering events can be filtered by the DKS method. We note that the discrimination capability based on the DKS method is higher for $14 \mathrm{MeV}$ TBN than $2.45 \mathrm{MeV}$ DDN events, which implies an even better singleto-noise ratio of TBN measurements. The proportion of the false $2.45 \mathrm{MeV}$ DDN events are significantly higher than that of $14 \mathrm{MeV}$ TBN, so the DKS method can give even better signal-to-noise ratio to measure the TBN for the helical plasmas. For the separate ring of the S2 scintillators, the range of proton energy deposited in S1 scintillators from the protons are different due to the different coverages of neutron scattering angles. The larger pulse height for the bottom ring is owing to the larger neutron scattering angles. By considering the finite geometry of S2, we expect that energies in the range Meanwhile, it can be learned that pulse heights of the effective events range from about $20 \mathrm{keVee}$ to $180 \mathrm{keVee}$ for the DDN, and from about $300 \mathrm{keVee}$ to $2300 \mathrm{keVee}$ for the TBN may be deposited in the S2 scintillators. It demands that the digitizersHence, there is need for in the DAQ system with a have dynamic range of 
TABLE III. The estimated neutron counting rates for DDN and TBN at the primary scintillators of the LHD-TOFED.

\begin{tabular}{|c|c|c|c|}
\hline \hline $\begin{array}{c}\text { Neutron } \\
\text { type }\end{array}$ & $\begin{array}{c}\text { Neutron Flux } \\
{\left[\mathrm{n} / \mathrm{s} \cdot \mathrm{cm}^{2}\right]}\end{array}$ & $\begin{array}{c}\text { Area efficiency } \\
{\left[\mathrm{cm}^{2}\right]}\end{array}$ & $\begin{array}{c}\text { Counting Rate } \\
{[\mathrm{kHz}]}\end{array}$ \\
\hline DDN & $10^{6}$ & 0.25 & 250 \\
TBN & $5 \cdot 10^{3}$ & 0.05 & 0.25 \\
\hline
\end{tabular}

3 orders of magnitude and with high signal-to-noise ratio which is necessary for the LHD-TOFED to measureto measured simultaneously the DDN and TBN emission simultaneouslywith the LHD-TOFED.

During the recentIn recent experimental campaigns at LHD, it was confirmed that the neutron yield ean reachreached more than $1 \times 10^{15} \mathrm{n} / \mathrm{s}$ and the TBN ratio ean be upwas up to $0.5 \%$. With the expected vertical-line-of sight, the neutron fluxes on S1 scintillators of the LHDTOFED are estimated to be $10^{6} \mathrm{n} / \mathrm{s}^{\cdot} \mathrm{cm}^{2}$ for DDN and $5 \cdot 10^{3}$ $\mathrm{n} / \mathrm{s} \cdot \mathrm{cm}^{2}$ for TBN. Considering the detection efficiency presented in TABLE III, the recorded counting rates of registere $\mathrm{DDN}$ and TBN could would be $250 \mathrm{kHz}$ and 250 $\mathrm{Hz}$, respectively ${ }_{5_{-}}$in which the count rates is possibly sufficient for the diagnosis of theThis will allow studies of the DDN and TBN emission on time scales of $1 / 10$ and some seconds for the two components, respectively. ratio. It demonstrates the feasibility of NES diagnosis by the LHD TOFED, and additionally, the neutron spectrometer might have the potentially application as the auxiliary diagnostic of TBN ratio on time scales of few seconds in the helical fusion plasmas at the LHD.

\section{CONCLUSION}

A TOFED-type neutron spectrometer (LHD-TOFED) is introduced and is under development at for the LHD and will, and it will be the first NES diagnostic in a helical/stellarator device. The main mission of the LHDTOFED is to measure the $2.5 \mathrm{MeV} \mathrm{D}$-D neutrons for diagnosing energetic ion behaviors in the-helical/stellarator plasmas, with the additional capability to measure $14 \mathrm{MeV}$ neutrons born in triton burn up reactions. Its capability of measuring the TBN is also evaluated. By using aBased on the digital data acquisition system and a double kinematic selection method, application of DKS method can well optimize the a sufficient signal-to-noise ratio for measuring both-simultaneously the DDN and TBN components can be obtained. It also demonstrates that the LHD-TOFED not only can be used for the diagnosis of fast deuterons, but alse indicates the potential as an auxiliary diagnostic for the $14 \mathrm{MeV}$ TBN ratio on time scales of few seconds in the helical plasmas at the LHD-during the typical experiment regime with high NBI power delivered.The LHD-TOFED will be one of the main diagnostics for studies of fast ions in deuterium helical plasmas at LHD and will also serve as a complementary diagnostics for investigations on the confinement of $\mathrm{MeV}$ range tritons born in DD fusion reactions.

\section{ACKNOWLEDGMENTS}

This work was supported by the National MCF Energy R\&D Program (Nos. 2019YFE03040000, 2013GB106004 and 2012GB101003), the National Key Research and Development Program of China (Nos. 2016YY0200805 and 2017YFF0206205), the State Key Program of National Natural Science of China (No. 11790324), and the User with Excellence Program of Hefei Science Center CAS (No. 2020HSC-UE012). The authors are very grateful to the EAST operation team for their help during the experimental campaigns.

'B. Wolle, Phys. Rep. 312, 1 (1999).

${ }^{2}$ G. Ericsson, L. Ballabio, S. Conroy, J. Frenje, H. Henriksson, A Hjalmarsson, J. Källne, and M. Tardocchi, Rev. Sci. Instrum. 72, 759 (2001).

${ }^{3}$ M. Gatu Johnson, L. Giacomelli, A. Hjalmarsson, J. Källne, M. Weiszflog, E. Andersson Sundén, S. Conroy, G. Ericsson, C. Hellesen, E. Ronchi, H. Sjöstrand, G. Gorini, M. Tardocchi, A. Combo, N. Cruz, J. Sousa, and S. Sjostrand, G. Gorini, M. Tardocchi, A. Combo, N. Cruz, J. Sousa, and S. Popovichev, Nucl. Instruments Methods Phys. Res. Sect. A Accel.
Spectrometers, Detect. Assoc. Equip. 591, 417 (2008).

${ }^{4}$ J. Källne, L. Ballabio, J. Frenje, S. Conroy, G. Ericsson, M. Tardocchi, E. Traneus, and G. Gorini, Phys. Rev. Lett. 85, 1246 (2000).

${ }^{5}$ C. Hellesen, M. Albergante, E.A. Sundén, L. Ballabio, S. Conroy, G. Ericsson, M.G. Johnsson, L. Giacomelli, G. Gorini, A. Hjalmarsson, Jenkins, J. Källne, E. Ronchi, H. Sjöstrand, M. Tardocchi, I Voitsekhovitch, M. Weiszflog, and J.-E. contributors, Plasma Phys. Control. Fusion 52, 085013 (2010).

${ }^{6}$ C. Hellesen, M. Gatu Johnson, E. Andersson Sundén, S. Conroy, G. Ericsson, J. Eriksson, H. Sjöstrand, M. Weiszflog, T. Johnson, G. Gorini, M. Nocente, M. Tardocchi, V.G. Kiptily, S.D. Pinches, and S.E. Sharapov, Nucl. Fusion 53, 113009 (2013).

${ }^{7}$ J. Eriksson, M. Nocente, F. Binda, C. Cazzaniga, S. Conroy, G. Ericsson,
L. J. Giacomelli, G. Gorini, C. Hellesen, T. Hellsten, A. Hjalmarsson, A.S. L. Giacomelli, G. Gorini, C. Hellesen, T. Hellsten, A. Hjalmarsson, A.S.
Jacobsen, T. Johnson, V. Kiptily, T. Koskela, M. Mantsinen, M. Salewski, M. Schneider, S. Sharapov, M. Skiba, M. Tardocchi, and M. Weiszflog, Nucl. Fusion 55, (2015).

${ }^{8}$ C. Hellesen, M. Mantsinen, S. Conroy, G. Ericsson, J. Eriksson, V.G. Kiptily, and F. Nabais, Nucl. Fusion 58, 123026 (2018).

${ }^{9}$ N.N. Gorelenkov, S.D. Pinches, and K. Toi, Nucl. Fusion 54, 125001 (2014).

${ }^{10}$ M. Nocente, J. Fusion Energy 38, 291 (2019).

${ }^{11}$ M. Osakabe, Y. Takeiri, T. Morisaki, G. Motojima, K. Ogawa, M. Isobe, M. Tanaka, S. Murakami, A. Shimizu, K. Nagaoka, H. Takahashi, K Nagasaki, H. Takahashi, T. Fujita, Y. Oya, M. Sakamoto, Y. Ueda, T. Nagasaki, H. Takahashi, T. Fujita, Y. Oya, M. Sakamoto, Y. Ueda, T.
Akiyama, H. Kasahara, S. Sakakibara, R. Sakamoto, M. Tokitani, H. Akiyama, H. Kasahara, S. Sakakibara, R. Sakamoto, M. Tokitani, H.
Yamada, M. Yokoyama, and Y. Yoshimura, Fusion Sci. Technol. 72, 199 (2017)

${ }^{12}$ M. Isobe, K. Ogawa, T. Nishitani, H. Miyake, T. Kobuchi, N. Pu, H. Kawase, E. Takada, T. Tanaka, S. Li, S. Yoshihashi, A. Uritani, J. Jo, S. Murakami, and M. Osakabe, IEEE Trans. Plasma Sci. 46, 2050 (2018).

${ }^{13}$ K. Ogawa, M. Isobe, T. Nishitani, R. Seki, H. Nuga, S. Murakami, M. Nakata, N. Pu, M. Osakabe, J. Jo, M. Cheon, J. Kim, G. Zhong, M. Xiao, and L. Hu, Plasma Phys. Control. Fusion 60, 095010 (2018).

${ }^{14} \mathrm{X}$. Zhang, Z. Chen, X. Peng, Z. Hu, T. Du, Z. Cui, X. Xie, X. Yuan, T. ${ }^{14}$ X. Zhang, Z. Chen, X. Peng, Z. Hu, T. Du, Z. Cui, X. Xie, X. Yuan, T.
Fan, J. Kallne, G. Gorini, M. Nocente, M. Tardocchi, L. Hu, G. Zhong, S. Lin, B. Wan, X. Li, G. Zhang, and J. Chen, Nucl. Fusion 54, 104008 (2014). ${ }^{15}$ X. Y. Peng, Z.J. Chen, X. Zhang, Z.M. Hu, T.F. Du, Z.Q. Cui, X.F. Xie,
. ${ }^{15}$ X.Y. Peng, Z.J. Chen, X. Zhang, Z.M. Hu, T.F. Du, Z.Q. Cui, X.F. Xie,
L.J. Ge, X. Yuan, G. Gorini, M. Nocente, M. Tardocchi, L.Q. Hu, G.Q. Zhong, S.Y. Lin, B.N. Wan, X.Q. Li, G.H. Zhang, J.X. Chen, and T.S. Fan, Rev. Sci. Instrum. 85, 11E112 (2014).

${ }^{16}$ X.Y. Peng, Z.J. Chen, X. Zhang, T.F. Du, Z.M. Hu, L.J. Ge, Y.M. Zhang, J.Q. Sun, G. Gorini, M. Nocente, M. Tardocchi, L.Q. Hu, G.Q. Zhong, N. Pu, S.Y. Lin, B.N. Wan, X.Q. Li, G.H. Zhang, J.X. Chen, and T.S. Fan, Rev. Sci. Instrum. 87, 11D836 (2016).

${ }^{17}$ L.J. Ge, Z.M. Hu, Y.M. Zhang, J.Q. Sun, X. Yuan, X.Y. Peng, Z.J. Chen, T.F. Du, M. Nocente, G. Gorini, M. Tardocchi, L.Q. Hu, G.Q. Zhong, B.N. Wan, X.Q. Li, and T.S. Fan, Rev. Sci. Instrum. 89, 101143 (2018). ${ }^{18} \mathrm{Y}$. Zhang, Ph.D. thesis, Peking University, in Chinese (2020). ${ }^{19}$ K. Ogawa, M. Isobe, T. Nishitani, S. Murakami, R. Seki, M. Nakata, E. Takada, H. Kawase, and N. Pu, Nucl. Fusion 58, 034002 (2018). 
${ }^{20} \mathrm{G}$. Gorini and J. Källne, Rev. Sci. Instrum. 63, 4548 (1992).

${ }^{21}$ X. Zhang, J. Källne, G. Gorini, M. Nocente, T. Fan, X. Yuan, X. Xie, and Z. Chen, Rev. Sci. Instrum. 85, 043503 (2014)

${ }^{2}$ Z.J. Chen, X.Y. Peng, X. Zhang, T.F. Du, Z.M. Hu, Z.Q. Cui, L.J. Ge, X.F. Xie, X. Yuan, G. Gorini, M. Nocente, M. Tardocchi, L.Q. Hu, G.Q. Zhong, S.Y. Lin, B.N. Wan, X.Q. Li, G.H. Zhang, J.X. Chen, and T.S. Fan, Rev. Sci. Instrum. 85, 11D830 (2014).

${ }^{23}$ M. Skiba, G. Ericsson, A. Hjalmarsson, C. Hellesen, S. Conroy, E. Andersson-Sundén, J. Eriksson, and JET Contributors, Nucl. Instruments Methods Phys. Res. Sect. A Accel. Spectrometers, Detect. Assoc. Equip. 833, 94 (2016)

${ }^{24}$ L.J. Ge, Z.M. Hu, Y.M. Zhang, J.Q. Sun, X. Yuan, X.Y. Peng, Z.J. Chen,

T.J. Ge, Z.M. Hu, Y.M. Zhang, J.Q. Sun, X. Yuan, X.Y.P Peng, Z.J.Chen,

T.F. Du, G. Gon X, M. Noch. Z. Lin, B.N. Wan, X.Q. Li, G.H. Zhang, J.X. Chen, and T.S. Fan, Plasma Phys. Control. Fusion 60, 095004 (2018).

${ }^{5}$ S. Agostinelli, Nucl. Instruments Methods Phys. Res. A 506, 250-303 (2003). 